\title{
Variación de la -/d/ final de palabra en Madrid: ¿prestigio abierto o encubierto?
}

\author{
Isabel Molina Martos ${ }^{1}$ \\ Universidad de Alcalá
}

\section{DE LA DIALECTOLOGÍA A LA SOCIOLINGÜÍSTICA: EL MARCO DE LA INVESTIGACIÓN}

Tradicionalmente, han dado noticia de la variación de la dental en coda dialectólogos y fonetistas como Navarro Tomás, quien, en su Manual de pronunciación española ( $1^{\mathrm{a}}$ ed. 1918), describía cómo se pronunciaba la dental sonora en final de palabra a comienzos del siglo XX, con algunos datos sobre la distribución geográfica y social de las variantes:

- $\left.\quad-d>{ }^{\delta}\right]:$ La $-d$ final absoluta, seguida de pausa, se articulaba particularmente débil y relajada incluso en pronunciación culta

- $\quad-\mathrm{d}>\left[{ }^{\delta}\right]$ : En palabras como sed, huésped, etc., los cultos conservaban siempre una $-d$ relajada

- $\quad-d>[\varnothing]$ En formas nominales, como virtud, verdad, etc., la pronunciación vulgar, en la mayor parte de España, suprimía la $d$ final. En Madrid, este uso se extendía incluso a la pronunciación familiar de las personas cultas.

\footnotetext{
Para correspondencia, dirigirse a: Isabel Molina Martos (isabel.molina@uah.es), Departamento de Filología, Comunicación y Documentación, Universidad de Alcalá, c/, Trinidad, 5, 28801- Alcalá de Henares - Madrid, España.
} 
- $\left.\quad-d>{ }^{\theta}\right]$ : En Valladolid, Salamanca y otros lugares de Castilla-León, en vez de una $-d$, se pronunciaba, como en coda silábica, una $\left.{ }^{\theta}\right]$ relajada y "lo mismo ocurre entre el pueblo bajo madrileño".

Desde la dialectología hispánica peninsular, se ha señalado que la pronunciación sorda o sonora de la $-d$ es uno de los rasgos fonéticos diferenciadores comúnmente conocidos "entre la Vieja y la Nueva Castilla". Efectivamente, en un estudio anterior comprobamos a través de la geografía lingüística que:

La articulación sorda se localiza fundamentalmente en tierras de Castilla, de forma más compacta en Burgos, Palencia, Valladolid, este de León y Zamora, norte de Segovia y Ávila, Soria; está presente, en menor proporción, en Logroño, Guadalajara, Cuenca y Madrid y se documenta escasamente en Toledo, Ciudad Real y Albacete. (García Mouton y Molina 2016: 280).

El cartografiado de las variantes de la -d final venía a probar, una vez más, que dialectalmente Madrid se encuentra en zona de transición dialectal al estar situado entre las hablas conservadoras del norte, que mantienen la dental y/o tienden a ensordecerla ([- $\left.\left.\delta,--^{-}, \theta\right]\right)$, y las hablas innovadoras del sur, Castilla La Mancha, Extremadura y Andalucía, que la relajan o la eliminan completamente $\left(\left[{ }^{-}, \varnothing\right]\right)$.

\section{OBJETIVOS DE LA INVESTIGACIÓN}

El objetivo general de esta investigación es presentar un análisis variacionista de la consonante dental en coda en la ciudad de Madrid. Este propósito se concreta en una serie de objetivos secundarios que se detallan a continuación:

Objetivo 1: analizar e interpretar las variantes en alternancia de la -d final de palabra, supuestamente sujetas a una estratificación social en la ciudad de Madrid. En este sentido, y desde un punto de vista dialectal, los madrileños tienen dos alternativas:

(1) Pueden optar por la conservación y/o el ensordecimiento en convergencia con las variedades castellanas del norte peninsular:

$$
-ð>-\theta
$$


(2) O bien pueden relajar la consonante hasta llegar a la elisión, un proceso que supone convergencia con las variedades meridionales.

$$
-ð>-^{ð}>\varnothing
$$

Pero la elección, además de convergencia dialectal, implica convergencia sociolingüística con determinados estratos sociales de la capital.

Objetivo 2: comprobar la evolución fónica del segmento dental a través del espacio léxico siguiendo el principio de la geografía lingüística según el cual "cada palabra tiene su propia historia".

Navarro Tomás (1971) advertía que para trazar la evolución de la dental en final de palabra es preciso observar la trayectoria de cada unidad léxica independientemente, pues "la verdadera unidad lingüística con geografía propia es la palabra [...]. La imagen semántica de cada palabra actúa sobre el tratamiento de sus sonidos" (apud. García Mouton y Molina 2016: 278).

Efectivamente, la realización de la dental en coda, por una parte, es un sonido relativamente poco frecuente en español y, por otra, la variedad de unidades léxicas en que aparece es muy limitada. Por ello, otro de los objetivos del análisis será establecer correlaciones entre unidad léxica y realización fonética.

El corpus de hablantes madrileños con el que se ha trabajado incluye más de 36 horas de grabación correspondientes a 36 informantes del barrio de Salamanca de Madrid y un total de 1459 realizaciones fonética de la -/d/, lo que posibilita estudiar el tratamiento que recibe la consonante caso por caso.

Objetivo 3: comparar la dinámica urbana del cambio con la rural A partir de los datos del Atlas Dialectal de Madrid (ADiM), García Mouton y Molina (2016) estudiaron la dinámica de la dental en final de palabra en las hablas rurales madrileñas. Los materiales que se recogieron para ese atlas posibilitan estudiar el cambio en tiempo aparente, a través de tres generaciones (García Mouton y Molina 2009), lo que permitió comprobar que, en las hablas rurales la dental en final de palabra había experimentado un cambio intergeneracional:

1. La TERCERA GENERACIÓN (55-75 años) del corpus rural representa la pronunciación tradicional: los hablantes mayores, tanto hombres como mujeres, alternan las tres variantes $[\varnothing],[ð],[\theta]$, pero en proporciones distintas: 
- $\quad[\varnothing]:$ los hombres favorecen la elisión [Ø]

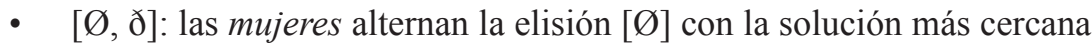
a la norma $[ð]$.

- $[\theta]$ : en unos y otras la proporción de la interdental sorda $[\theta]$ es baja.

2. En la SEGUNDA GENERACIÓN (35-54 años) la cartografía de la -d revela la introducción de una innovación fónica: los hombres de esta generación han sustituido la elisión por la dental sorda $[\theta]$ y las mujeres, aunque por detrás, han evolucionado en el mismo sentido.

3. PRIMERA GENERACIÓN (20-34 años): por último, los hombres más jóvenes consolidan el cambio que inició la generación de sus padres con un comportamiento casi idéntico $([\theta])$; por su parte, las mujeres jóvenes difieren de los hombres de su edad retrocediendo un poco respecto a la segunda generación y volviendo a inclinarse por la variante sonora [ð]. Se puede concluir que, aunque los jóvenes apoyaron la innovación que habían introducido sus padres, el cambio, en lugar de consolidarse, experimentó un cierto retroceso.

En síntesis, puede afirmarse que en las hablas rurales de Madrid la $-\mathrm{d}$ experimentó un cambio muy evidente entre la $3^{\mathrm{a}}$ y la $2^{\mathrm{a}}$ generación: $[\varnothing]>$ $[\theta]$, un cambio que los jóvenes apoyaron con reservas. Así, en el paso de la $2^{\mathrm{a}}$ a la $1^{\mathrm{a}}$ generación, la difusión de la variante sorda sufrió un retroceso, más acusado en las mujeres que en los hombres.

\section{METODOLOGÍA: EL BARRIO DE SALAMANCA DE MADRID: EL CORPUS DE DATOS Y LA MUESTRA SOCIOLINGÜÍSTICA}

Para este estudio se ha analizado un corpus recogido en el marco del Proyecto para el Estudio Sociolingüístico del Español de España y América (PRESEEA) para el barrio de Salamanca de Madrid. Se han estudiado las realizaciones de la dental en una muestra de 36 informantes preestratificada por edad, sexo y nivel de instrucción, y postestratificada por clase social. Si la preestratificación por cuotas fijas asegura un mismo número de hombres y mujeres de cada nivel de instrucción y de cada grupo de edad, la postestratificación revela que solo uno de los informantes pertenecía a la clase baja, dos a la clase media-baja y el resto a las clases media y mediaalta. El habla representada en este corpus es exponente, por tanto, de los estratos sociales medio y medio-alto de la ciudad. 
Se han transcrito aproximadamente 36 horas de entrevistas semidirigidas, que han arrojado 1450 realizaciones fonéticas del segmento dental sonoro en final de palabra. El gráfico 1 muestra cómo en el barrio de Salamanca la dental en coda se realiza con tres clases de variantes:

- [ð] fricativa/aproximante, dental, sonora, con distintos grados de relajación

- $[\varnothing]$ elisión

- $[\theta]$ dental fricativa sorda, con distintos grados de relajación

De las tres clases de variantes registradas, la elisión es la más frecuente; muy por detrás siguen las dentales sonora y sorda.

Gráfico 1: variantes de -/d/ en el barrio de Salamanca

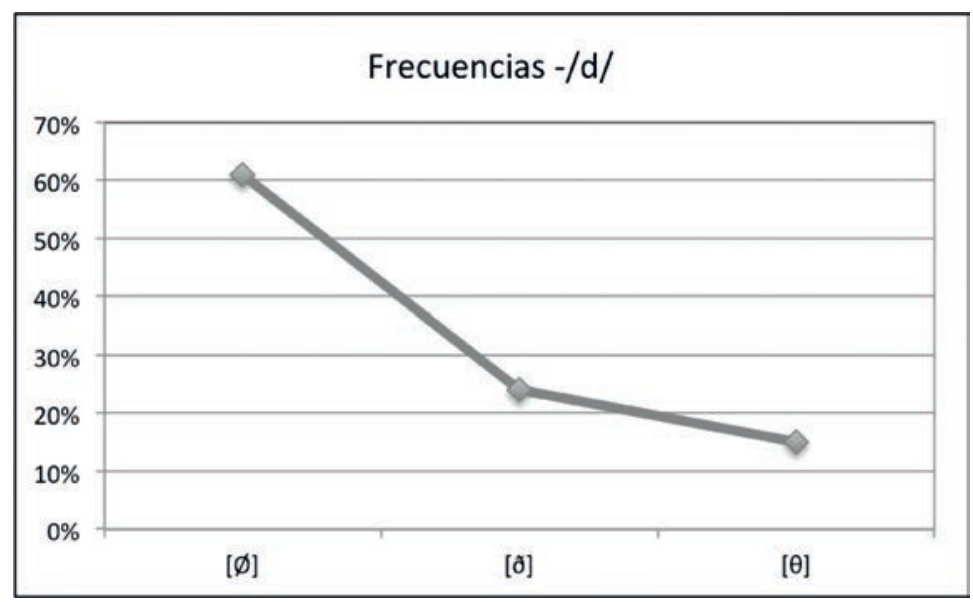

EL ANÁLISIS LOGÍSTICO DE REGRESIÓN

Para este estudio sociolingüístico se hizo con el programa Goldvarb $\mathrm{X}^{2}$ un análisis logístico de regresión para medir la incidencia de factores lingüísticos, estilísticos y sociales en la realización de las distintas variantes

2 Programa estadístico de acceso libre creado por los investigadores de las universidades de Toronto y Ottawa Sankoff y Rousseau (1979); vid. también Sankoff, Tagliamonte y Smith (2012); está diseñado específicamente para el análisis de datos lingüísticos. 
de -/d/. Entre las posibilidades que permite el programa, se ha optado por un análisis de regresión escalonado de subida y bajada (up\&down), que determina la incidencia conjunta de las variables sobre cada una de las tres clases de variantes fonéticas. El programa selecciona el conjunto de variables de mayor capacidad explicativa del fenómeno analizado, a la vez que señala cuáles quedan descartadas por su menor contribución a la explicación de la variable ${ }^{3}$.

\section{EL ANÁLISIS DE CORRELACIONES BIVARIANTES}

El análisis logístico de regresión se complementó con un análisis de correlaciones bivariantes realizado con SPSS, que establece si existe incidencia o correlación entre cada una de las variables independientes sobre la variable dependiente aplicando las pruebas de $j i$ cuadrado $\left(\chi^{2}\right)$ y $\mathrm{V}$ de Cramer.

\section{VARIABLES INDEPENDIENTES}

Se ha medido la posible incidencia sobre la realización de la -/d/ en coda y final de palabra de cinco factores lingüiśticos:

1. Entorno fonológico siguiente: vocal, consonante, pausa

2. Vocal previa: $[\mathrm{a}, \mathrm{e}, \mathrm{i}, \mathrm{o}, \mathrm{u}]$

3. Unidad léxica: Madrid, usted, verdad, edad, ciudad, otras.

4. Categoría léxica: nombre común, nombre propio

5. Número de sílabas de la palabra: bisílaba, polisílaba.

Un solo factor estilístico: la fase de la interacción: comienzo, intermedio y final.

Y cinco factores sociales, tres de preestratificación, que siguen la metodología propuesta por PRESEEA, y un factor de postestratificación, la clase social, determinada por el equipo PRESEEA-Madrid a partir de la suma paramétrica de la profesión y el nivel de ingresos:

3 Para determinar la bondad de la prueba se usa la razón de verosimilitud (log likelihood), una prueba equivalente al $j i$ cuadrado. Cuanto más cerca de 0 esté el valor del estadístico, más capacidad explicativa tiene la variable. En todo caso, siempre hay que tener en cuenta el nivel de significación que queremos otorgar a nuestro análisis, que convencionalmente ha de ser igual o menor de 0,05 en los estudios de ciencias sociales. 
1. Sexo

2. Edad ( $1^{\mathrm{a}}$ generación $20-34$ años; $2^{\mathrm{a}}$ generación $35-54$ años; $3^{\mathrm{a}}$ generación más de 55 años);

3. Nivel de instrucción (estudios primarios; secundarios; universitarios);

4. Clase social (baja, media-baja, media, media-alta);

\section{EL ANÁLISIS}

Se realizaron tres análisis logísticos de regresión, uno por cada una de las tres clases de variantes. En la tabla 1 se sintetizan los datos generales de los tres análisis:

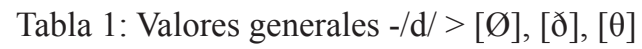

\begin{tabular}{|c|c|c|c|}
\hline & {$[\varnothing]$} & {$[\varnothing]$} & {$[\theta]$} \\
\hline INPUT & 0.631 & 0.209 & 0.113 \\
\hline SigNIFICACIÓN & 0.040 & 0.010 & 0.007 \\
\hline LOG PROBABILID & -844.067 & -720.930 & -527.694 \\
\hline FRECUENCIAS & $60,9 \%(889)$ & $24,1 \%(351)$ & $15 \%(219)$ \\
\hline
\end{tabular}

En la realización de las variantes inciden tres de las cinco variables lingüísticas que se sometieron a análisis: el "entorno fonológico siguiente", la "vocal previa", y la "unidad léxica" en que aparece la dental (Madrid, usted, verdad, edad, ciudad, otras); en cambio, parece tener muy poco poder explicativo que el nombre sea común o propio, o que la palabra tenga dos o más sílabas. A continuación se presentan los datos de estadística inferencial de cada una de las variables que sí resultaron significativas:

\section{(1) ENTORNo FONOLÓGICO SIGUIENTE}

Tabla 2: Variantes de -/d/ y entorno fonológico siguiente

\begin{tabular}{|l|c|c|c|}
\hline & {$[\varnothing]$} & {$[ð]$} & {$[\theta]$} \\
\hline PAUSA & 0.459 & $\mathbf{0 , 5 8 0}$ & 0.455 \\
\hline CONSONANTE & $\mathbf{0 . 6 2 6}$ & 0.408 & 0.409 \\
\hline VOCAL & 0.374 & 0.510 & $\mathbf{0 . 7 0 4}$ \\
\hline
\end{tabular}


Gráfico 2. Variantes de -/d/ y entorno fonológico siguiente

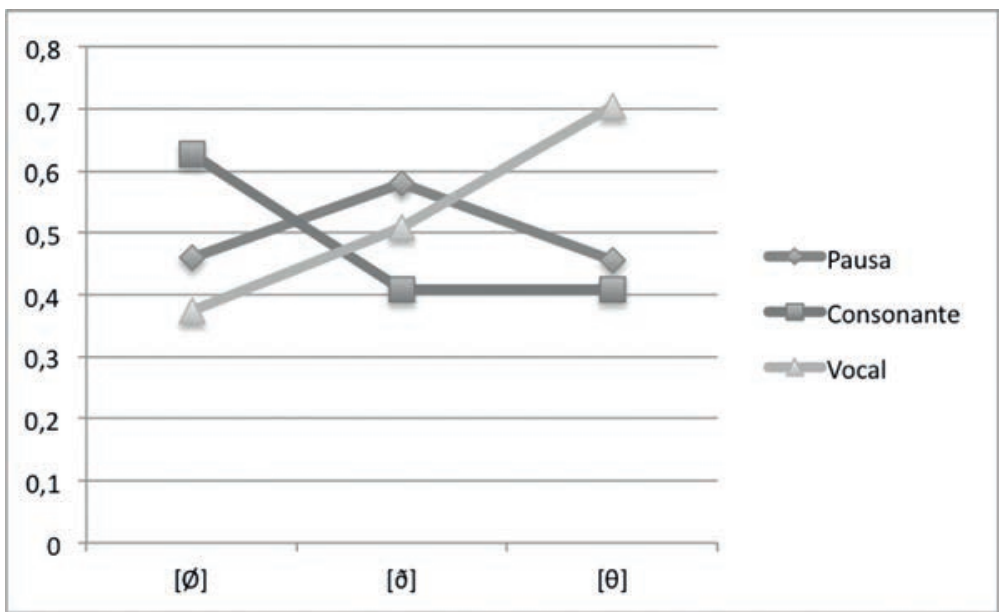

El entorno fonológico que sigue a la dental explica, en parte, la aparición de cada una de las variantes: la dental tiende a elidirse cuando va seguida de una consonante; a realizarse como dental sonora cuando va seguida de pausa, y a ensordecerse cuando le sigue una vocal.

(2) Vocal PREVIa

Tabla 3: Variantes de -/d/ y vocal previa

\begin{tabular}{|c|c|c|c|}
\hline & {$[\varnothing]$} & {$[ð]$} & {$[\theta]$} \\
\hline$[\mathrm{e}]$ & 0,283 & & $\mathbf{0 , 6 7 4}$ \\
\hline$[\mathrm{a}]$ & $\mathbf{0 , 6 2 6}$ & & 0,383 \\
\hline$[\mathrm{i}]$ & 0,429 & & 0,599 \\
\hline$[\mathrm{o}, \mathrm{u}]$ & 0,250 & & $\mathbf{0 , 6 9 4}$ \\
\hline
\end{tabular}


Gráfico 3. Variantes de -/d/ y vocal previa

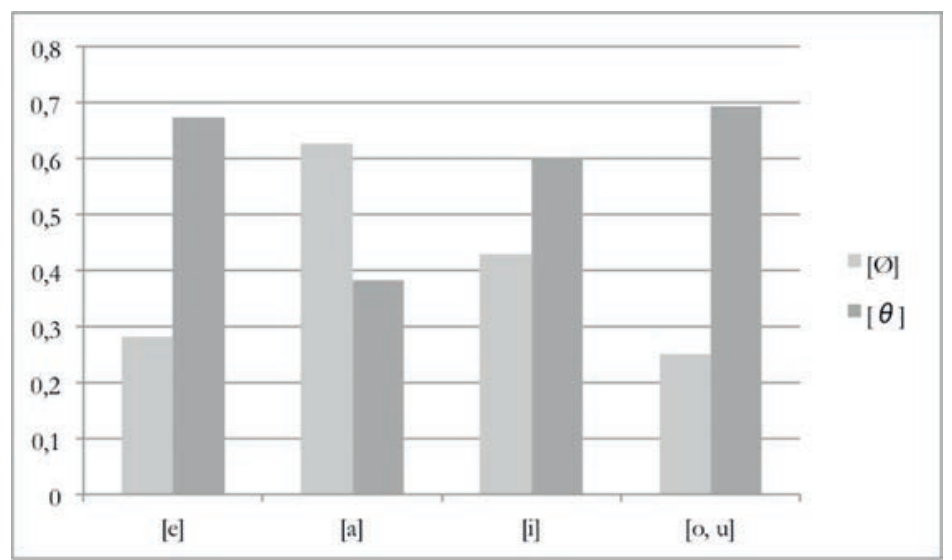

El análisis determinó que cuál sea la vocal que precede a la dental es determinante solo para dos de las variantes: la elisión y la dental sorda. La dental se elide principalmente cuando va precedida de la [a] (la verdá es $q u e)$, y se ensordece si va precedida de [e, o, u] (ustez, saluz, virtuz).

(3) UNIDAD LÉXICA

Tabla 4: variantes de -/d/ y unidad léxica

\begin{tabular}{|l|c|c|c|}
\hline & {$[\varnothing]$} & {$[\varnothing]$} & {$[\theta]$} \\
\hline usted & 0.545 & 0.568 & 0.467 \\
\hline edad & 0.125 & 0.660 & 0.827 \\
\hline Madrid & 0.484 & 0.605 & 0.318 \\
\hline otras & 0.527 & 0.534 & 0.535 \\
\hline ciudad & 0.368 & 0.206 & 0.796 \\
\hline verdad & 0.585 & 0.234 & 0.546 \\
\hline
\end{tabular}


Gráfico 4: variantes de -/d/ y unidad léxica

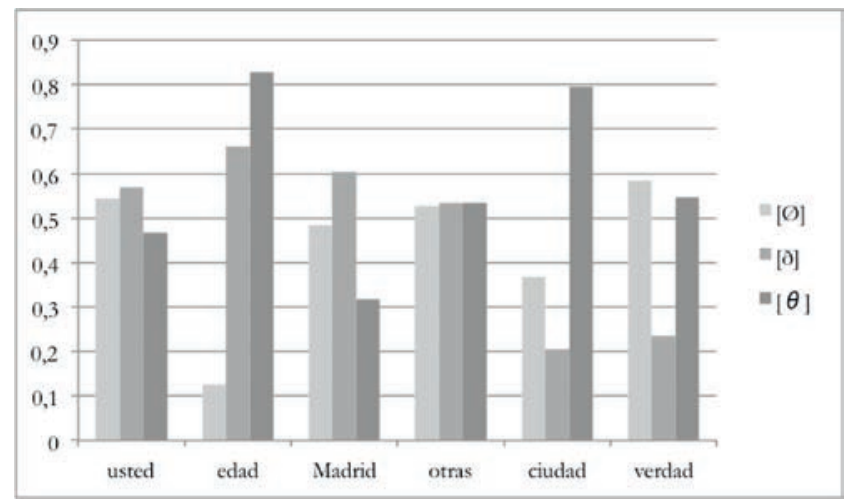

En el corpus del barrio de Salamanca, la solución más general es la elisión, que alterna con las otras dos variantes; sin embargo, ciertas unidades léxicas, pese a ser susceptibles de realizarse con cualquiera de las tres variantes, favorecen alguna de ellas:

[Ø]: verdad > verdá

[ð]: Madrid, usted $>$ Madrid, usted

[0]: edad, ciudad > edaz, ciudaz

Según se verá más adelante, la variable -/d/ mantiene un equilibrio relativamente estable en el barrio de Salamanca.

El único de los factores estilísticos que se sometió a análisis, la "fase de la interacción", tampoco resultó significativo. Se trataba de saber si, a medida que avanza la entrevista semidirigida y el hablante se relaja, produce con más frecuencia alguna de las tres variantes, como se ha comprobado que sucede en Madrid con algunas variables fónicas como la caída de la-den posición intervocálica o la lenición de la $-\mathrm{s}$ en coda (Molina y Paredes 2014, Molina 2015). Que la dental no tenga valor estilístico supone que los hablantes no la emplean para marcar su discurso como más o menos formal o coloquial, y sin embargo sí parece haber dentro de la comunidad conciencia lingüística de las distintas variantes. La ausencia de valor estilístico podría indicar que la innovación, de estar produciéndose, o bien se encuentra en una fase inicial del proceso, o bien se mantiene relativamente inactiva en la comunidad objeto de estudio. 


\section{FACTORES SOCIALES}

El análisis de los factores sociales, aporta mucha información relevante para explicar por qué en el barrio de Salamanca la variable que estudiamos no muestra gran actividad. Veamos cómo se estratifican las variantes en la comunidad madrileña:

\section{(1) SEXo}

Tabla 5: variantes de -/d/ y sexo

\begin{tabular}{|l|c|c|c|}
\hline & {$[\varnothing]$} & {$[ð]$} & {$[\theta]$} \\
\hline Hombre & & $\mathbf{0 . 5 4 8}$ & 0.421 \\
\hline MuJer & & 0.453 & $\mathbf{0 . 5 7 7}$ \\
\hline
\end{tabular}

Los hombres y las mujeres solo muestran comportamiento distintos en la realización de $[ð]$ y $[\theta]$, presumiblemente porque la elisión es la solución general entre ellos y unos y otras la emplean indistintamente. En cambio, sí muestran preferencias distintas ante las soluciones minoritarias: los hombres prefieren la dental sonora $[ð]$ y las mujeres la sorda $[\theta]$.

Gráfico 5. Variantes de -/d/ y sexo

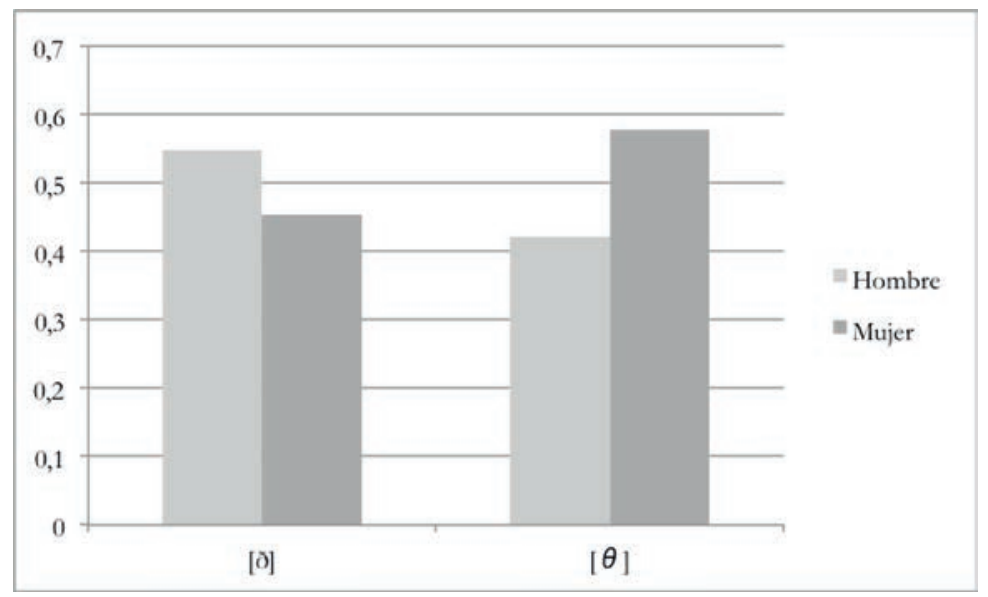


(2) EDAD

Tabla 6: variantes de -/d/ y edad

\begin{tabular}{|l|l|l|l|}
\hline & {$[\varnothing]$} & {$[ð]$} & {$[\theta]$} \\
\hline $1^{\mathrm{a}}$ GENERAC & 0.425 & $\mathbf{0 . 6 0 5}$ & 0.479 \\
\hline $2^{\mathrm{a}}$ GENERAC & 0.420 & 0.502 & $\mathbf{0 . 6 2 7}$ \\
\hline $3^{\mathrm{a}}$ GENERAC & $\mathbf{0 . 7 0 3}$ & 0.359 & 0.349 \\
\hline
\end{tabular}

Gráfico 6. Variantes de -/d/ y edad

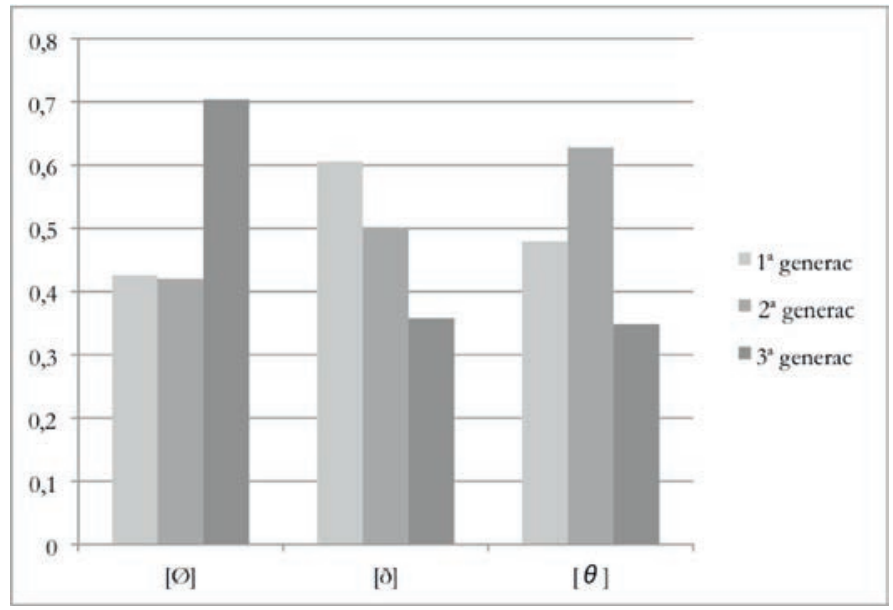

La estratificación por edad revela distintas preferencias generacionales: la solución elegida por los mayores es la elisión [Ø]; la segunda generación favorece el ensordecimiento $[\theta]$ y los jóvenes retoman la variante normativa [ð]. Obsérvese que en el barrio de Salamanca se percibe la misma estratificación intergeneracional que en las hablas rurales en la elección de las variantes, si bien a mucha menor escala en la ciudad. Como en los pueblos, en el barrio de Salamanca el desarrollo a través de las tres generaciones no ha sido lineal. Si los mayores emplean la elisión que tradicionalmente ha sido característica de la comunidad madrileña, entre 1970-1990, cuando los hablantes de la segunda generación tenían en torno a 20 años, se alejaron de la solución meridional $[\varnothing]$ para acercarse a la del norte $[\theta]$; la generación siguiente, la de los jóvenes, no imitó la innovación y prefirió, en cambio, la pronunciación normativa [ð]. Como puede observarse, se trata de un mismo desarrollo intergeneracional: 


\section{$3^{\mathrm{a}}$ gen $[\varnothing]>2^{\mathrm{a}}$ gen $[\boldsymbol{\theta}]>1^{\mathrm{a}} \operatorname{gen}[\boldsymbol{\delta}]$}

Pese a compartir la secuenciación intergeneracional de las variantes, se detectan importantes diferencias entre campo y ciudad: en el barrio de Salamanca la baja frecuencia de las variantes conservadoras indica que la innovación solo penetró tímidamente en las mujeres de la segunda generación pero rápidamente se retrajo y no pasó a la generación más joven. En las hablas rurales, por el contrario, prendió con mucha fuerza tanto en los hombres como en las mujeres de la segunda generación -más en ellos que en ellas-, y experimentó una cierta retracción entre los jóvenes, mucho más acusada en las mujeres, pues los hombres han sido los líderes indudables de un proceso con prestigio encubierto.

\section{(3) NiVEL DE INSTRUCCIÓN}

Tabla 7: variantes de -/d/ y nivel de instrucción

\begin{tabular}{|l|c|c|c|}
\hline & {$[Ø]$} & {$[ð]$} & {$[\theta]$} \\
\hline PRIMARIOS & 0.466 & 0.414 & $\mathbf{0 . 6 4 4}$ \\
\hline SECUNDARIOS & 0.449 & $\mathbf{0 . 6 0 5}$ & 0.439 \\
\hline UNIVERSITARIOS & $\mathbf{0 . 5 5 8}$ & 0.484 & 0.443 \\
\hline
\end{tabular}

Gráfico 7: variantes de -/d/ y nivel de instrucción

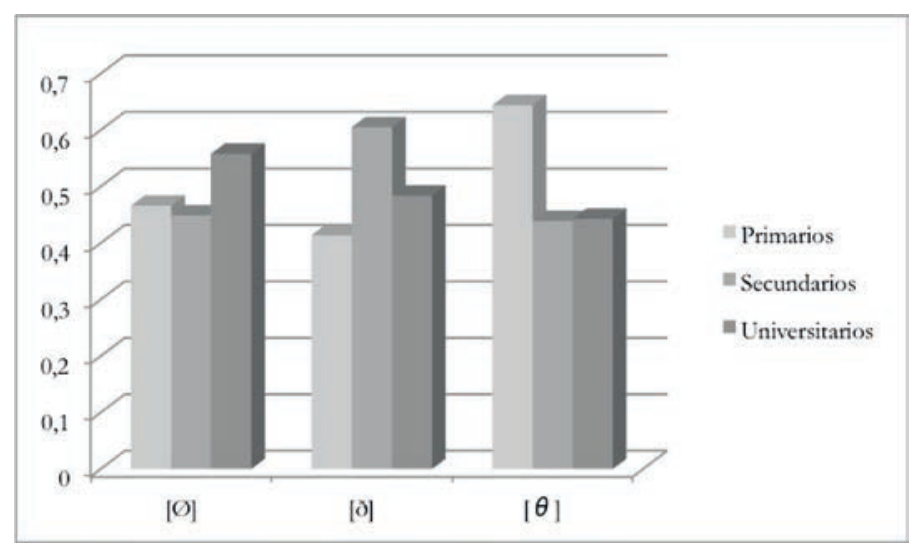


El nivel de instrucción, como la edad, también sigue pautas complementarias: parece que son los universitarios los que prefieren la elisión característica de la comunidad [Ø], mientras que los hablantes con estudios secundarios optan por la forma normativa [ð], y los de estudios primarios por la fricativa sorda $[\theta]$. Esta preferencia de los niveles de instrucción más bajos por la $z$ final fue retomada en los años de la Transición (1975-1982) como parte de la cultura marginal que se popularizaba entonces; en la década de 1980 la forma Madriz fue uno de los muchos exponentes de la movida madrileña y del origen popular de algunas de sus manifestaciones.

\section{(4) Clase social}

Tabla 8: variantes de -/d/ y clase social

\begin{tabular}{|l|c|c|c|}
\hline & {$[\varnothing]$} & {$[ð]$} & {$[\theta]$} \\
\hline BAJA & 0.426 & 0.685 & 0.462 \\
\hline MEDIA-BAJA & 0.757 & 0.394 & 0.244 \\
\hline MEDIA & 0.400 & $\mathbf{0 . 5 7 8}$ & $\mathbf{0 . 5 6 3}$ \\
\hline MEDIA-AltA & $\mathbf{0 . 6 2 2}$ & 0.359 & 0.464 \\
\hline
\end{tabular}

Para explicar estos datos se dejarán a un lado las clases baja y media-baja porque en la muestra solo incluyen uno y dos hablantes respectivamente, mientras que la gran mayoría se concentra en la clase media y en la mediaalta, que es donde necesariamente tendrá más interés acercarse a los resultados.

Gráfico 8: variantes de -/d/ y clase social

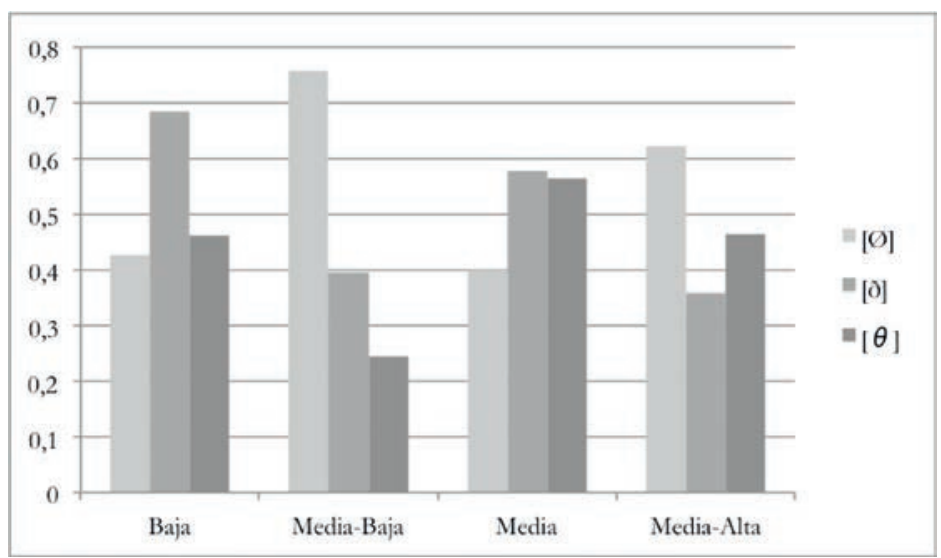


Efectivamente, la clase media-alta -igual que los universitarios-adopta la elisión como solución preferida, mientras que las clases medias optan por las dos variantes conservadoras, siguiendo una pauta de hipercorrección que suprime la elisión meridional y la sustituye por formas que conectan con el norte peninsular, cuyas variedades tienen más prestigio lingüístico en Madrid que las variedades meridionales.

La aplicación de las correlaciones bivariantes con SPSS permite combinar las variables sociales y, con ello, afinar un poco más en la estratificación sociolingüística de la dental en el barrio de Salamanca y en el perfil social de los hablantes que favorecen una y otra variante.

Tabla 9./d/ + sexo + edad -/d/ + sexo + edad

\begin{tabular}{|c|c|c|c|c|}
\hline \multirow[t]{2}{*}{ CORRELACIÓN } & \multirow[t]{2}{*}{ EDAD } & \multirow[t]{2}{*}{ VARIANTES -D } & \multicolumn{2}{|c|}{ SeXo } \\
\hline & & & Hombre & MUJER \\
\hline \multirow{3}{*}{$\begin{array}{l}\chi^{2}=41,280(2) \\
p=0,000 \\
\text { V de Cramer: } 0,281 \\
p=0.000\end{array}$} & \multirow{3}{*}{$1^{\mathrm{a}}$ GENERACIÓN } & [Ø] & $\mathbf{7 1 , 5} \%(171)$ & $43,8 \%(124)$ \\
\hline & & [ð] & $18,8 \%(45)$ & $40,6 \%(115)$ \\
\hline & & {$[\theta]$} & $9,6 \%(23)$ & $15,5 \%(44)$ \\
\hline \multirow{3}{*}{$\begin{array}{l}\chi^{2}=48,706(3) \\
p=0,000 \\
V \text { de Cramer: } 0,298 \\
p=0.000\end{array}$} & \multirow[t]{3}{*}{$2^{\mathrm{a}}$ GENERACIÓN } & {$[\varnothing]$} & $59,6 \%(165)$ & $54,8 \%(149)$ \\
\hline & & [ð] & $29,6 \%(82)$ & $13,2 \%(36)$ \\
\hline & & {$[\theta]$} & $10,5 \%(29)$ & $32 \%(87)$ \\
\hline \multirow{3}{*}{$\begin{array}{l}\chi^{2}=11,053(2) \\
p=0,004 \\
\text { V de Cramer: } 0,169 \\
p=0.004\end{array}$} & \multirow{3}{*}{$3^{\mathrm{a}}$ GENERACIÓN } & [Ø] & 67,2\% (137) & $77,2 \%(142)$ \\
\hline & & [ð] & $25 \%(51)$ & $12 \%(22)$ \\
\hline & & {$[\theta]$} & $7,8 \%(16)$ & $10,9 \%(20)$ \\
\hline
\end{tabular}

Si se cruzan las variables "sexo" y "edad", puede verse que los hombres de todas las generaciones seleccionan en primer lugar la elisión, mientras que entre las mujeres las diferencias intergeneracionales son más acusadas: las mayores también eligen la elisión, pero las de edad intermedia incorporan la variante sorda y las jóvenes la sonora. Así pues, son ellas quienes, aunque tímidamente, reproducen la convergencia con las variedades del norte y la divergencia con las del sur, pero este apoyo a la tendencia innovadora no se hace de manera decidida, presumiblemente porque no cuenta con el prestigio suficiente en la comunidad, ni abierto ni encubierto, tal y como queda reflejado en el gráfico siguiente: 


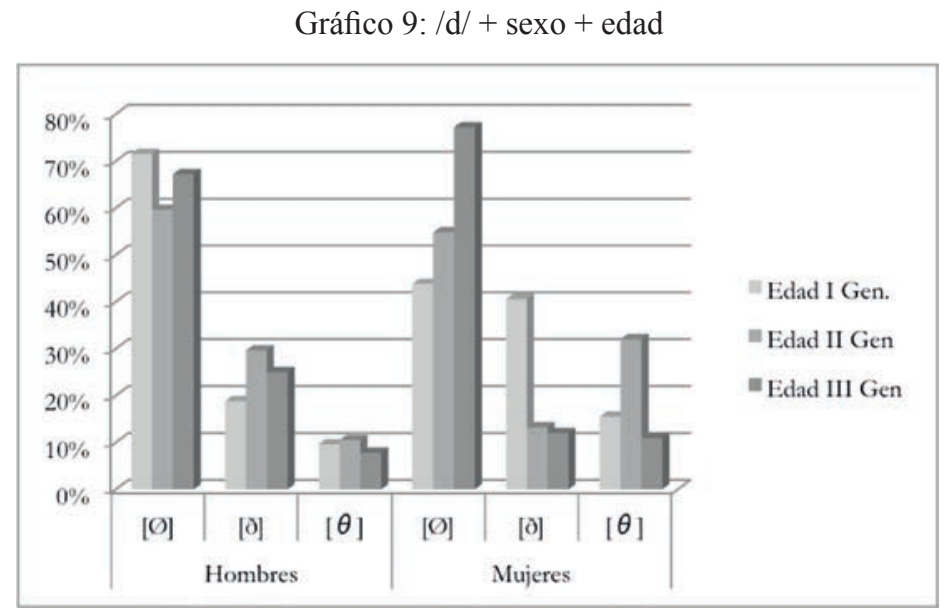

Tabla 10: sexo y clase social -/d/ + sexo + clase social

\begin{tabular}{|c|c|c|c|c|}
\hline \multirow[t]{2}{*}{ CORRELACIÓN } & \multirow[t]{2}{*}{ Clase social } & \multirow[t]{2}{*}{ VARIANTES -D } & \multicolumn{2}{|c|}{ SEXO } \\
\hline & & & НомBRE & MUJER \\
\hline & \multirow{3}{*}{ BAJA } & [Ø] & & $71,1 \%(27)$ \\
\hline & & [ð] & & $15,8 \%(6)$ \\
\hline & & {$[\theta]$} & & $13,2 \%(5)$ \\
\hline & \multirow{3}{*}{ Media-BAJA } & [Ø] & $72,8 \%(98)$ & \\
\hline & & {$[ð]$} & $18,5 \%(25)$ & \\
\hline & & {$[\theta]$} & $8,9 \%(12)$ & \\
\hline \multirow{3}{*}{$\begin{array}{l}\chi^{2}=54,414(2) \\
p=0,000 \\
\text { V de Cramer: } 0,251 \\
p=0.000\end{array}$} & \multirow{3}{*}{ MEdIA } & [Ø] & $63,1 \%(200)$ & $45,5 \%(250)$ \\
\hline & & {$[ð]$} & $30,6 \%(97)$ & $28,2 \%(155)$ \\
\hline & & {$[\theta]$} & $6,3 \%(20)$ & $26,2 \%(144)$ \\
\hline \multirow{3}{*}{$\begin{array}{l}\chi^{2}=34,050(2) \\
p=0,000 \\
\text { V de Cramer: } 0,285 \\
p=0.000\end{array}$} & \multirow{3}{*}{ Media-Alta } & [Ø] & $65,7 \%(176)$ & $\mathbf{9 0 , 8} \%(138)$ \\
\hline & & [ð] & $20,9 \%(56)$ & $7,9 \%(12)$ \\
\hline & & {$[\theta]$} & $13,4 \%(36)$ & $1,3 \%(2)$ \\
\hline
\end{tabular}


Gráfico 10: sexo y clase social

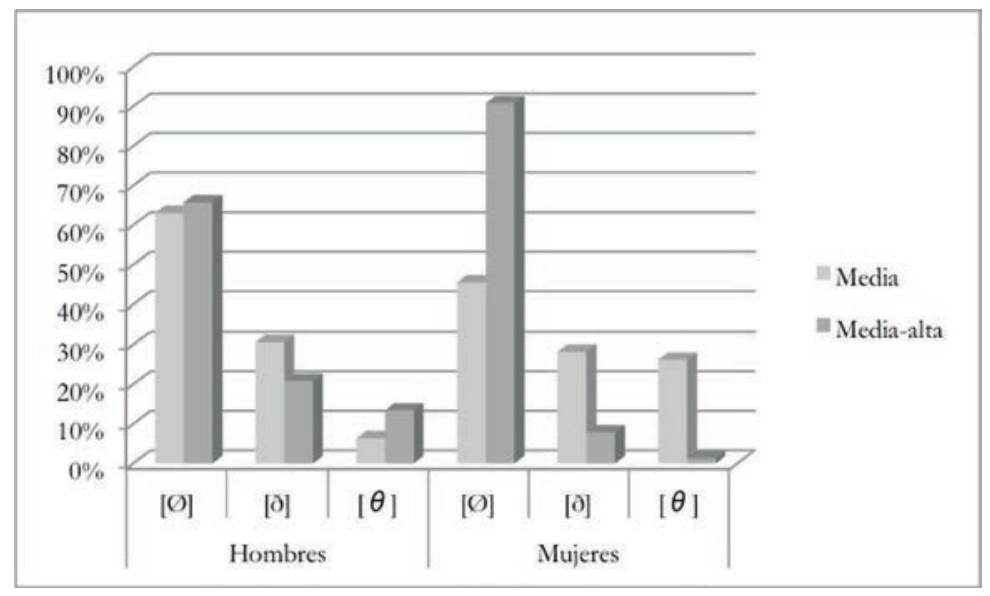

En el gráfico 10 solo se representan las clases media y media-alta por las razones que se indicaron más arriba. El gráfico muestra claramente que, tanto en hombres como en mujeres, la variante preferida es la elisión; de todos ellos, quienes más la emplean son las mujeres de clase media-alta, con valores cercanos al 90\%. Por el contrario, esas mismas mujeres, como el conjunto de los hombres, prácticamente nunca ensordecen la dental, lo que corrobora nuestra apreciación de que la variante no goza en el barrio de ninguna forma de prestigio, ni abierto ni encubierto.

Tabla 11: -/d/ + instrucción + clase social -/d/

\begin{tabular}{|c|c|c|c|c|c|}
\hline \multirow{2}{*}{ CORRELACIÓN } & \multirow{2}{*}{$\begin{array}{l}\text { Clase } \\
\text { SOCIAL }\end{array}$} & \multirow{2}{*}{$\begin{array}{c}\text { VARIANTES } \\
\text {-D }\end{array}$} & \multicolumn{3}{|c|}{ NIVEL INSTRUCCIÓN } \\
\hline & & & PRIMARIA & Secund. & UNIVERS. \\
\hline & \multirow{3}{*}{ BAJA } & {$[\varnothing]$} & $71,1 \%(27)$ & & \\
\hline & & [ð] & $15,8 \%(6)$ & & \\
\hline & & {$[\theta]$} & $13,2 \%(5)$ & & \\
\hline & \multirow{3}{*}{ Media-BAJA } & {$[\varnothing]$} & $71,9 \%(97)$ & & \\
\hline & & [ð] & $18,5 \%(25)$ & & \\
\hline & & {$[\theta]$} & $8,9 \%(12)$ & & \\
\hline
\end{tabular}




\begin{tabular}{|c|c|c|c|c|c|}
\hline$\chi^{2}=25,099$ & \multirow[b]{3}{*}{ Media } & {$[\varnothing]$} & $50,6 \%(122)$ & $52,2 \%(210)$ & $52,9 \%(118)$ \\
\hline$(4)$ & & [ð] & $20,7 \%(50)$ & $31,8 \%(128)$ & $33,2 \%(74)$ \\
\hline $\begin{array}{l}\mathrm{p}=0,000 \\
\mathrm{~V} \text { de Cramer: } \\
0,120 \\
\mathrm{p}=0.000\end{array}$ & & {$[\theta]$} & $28,6 \%(69)$ & $15,9 \%(64)$ & $13,9 \%(31)$ \\
\hline \multirow{3}{*}{$\begin{array}{l}\chi^{2}=67,469(2) ; \\
p=0,000 ; \\
V \text { de Cramer: } \\
0,401, \\
p=0.000\end{array}$} & \multirow[b]{3}{*}{ Media-Alta } & [Ø] & & $25 \%(7)$ & $\mathbf{7 8 , 3} \%(307)$ \\
\hline & & [ð] & & $71,4 \%(20)$ & $12,2 \%(48)$ \\
\hline & & {$[\theta]$} & & $3,6 \%(1)$ & $9,4 \%(37)$ \\
\hline
\end{tabular}

El gráfico 11 confirma la afirmación anterior: la clase media-alta y los universitarios prefieren, muy por delante del resto de las variantes, la elisión, que se revela como la forma con prestigio de grupo en el barrio de Salamanca; en el otro extremo de frecuencia, la variante sorda no se emplea prácticamente nunca. La clase media reproduce esta tendencia con algunos matices: utiliza todas las variantes pero con una estratificación social que prefiere, por este orden: la elisión, seguida de la dental sonora y por último de la sorda, solo defendida por los hablantes de instrucción primaria.

Gráfico 11:-/d/ + instrucción + clase social

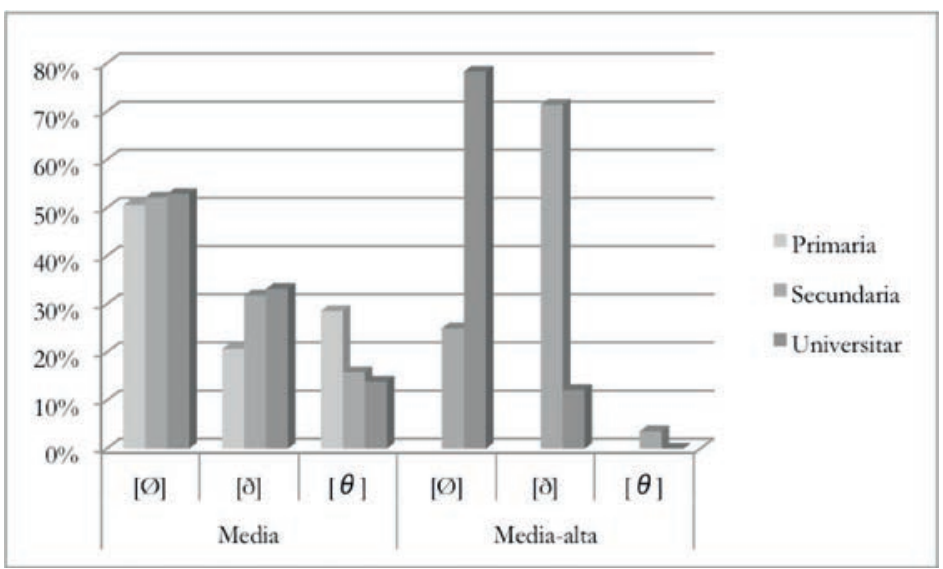




\section{CONCLUSIONES}

El análisis de la variable nos ha permitido conocer el significado social de las tres variantes de la -/d/ final de palabra en el barrio de Salamanca de Madrid:

La elisión $[\varnothing]$ es la solución característica de la comunidad de habla madrileña con prestigio encubierto en el barrio de Salamanca, preferida por las clases medias altas y por los universitarios. La interdental sorda [ $\theta]$ es la variante con prestigio encubierto en los niveles populares de Madrid, que intentaron un cambio desde abajo sin éxito en el barrio de Salamanca. Por último, la dental sonora $[ð]$ tiene prestigio abierto en la ciudad y es la preferida por las generaciones jóvenes.

Desde el punto de vista social, las clases medias altas, los hablantes cultos y los mayores optan por la elisión, una variante que, pese a ser meridional, mantiene su prestigio en los niveles altos del barrio de Salamanca. La elisión representa la pronunciación tradicional en el área dialectal en que se inscribe la comunidad madrileña, y así como en los estratos altos las otras consonantes en coda -como la -/s/- han retrocedido en el proceso de lenición siguiendo la norma castellana, no sucede lo mismo con la elisión de - d en final de palabra, que goza de prestigio encubierto en la comunidad, tal vez por su aparición limitada a unas pocas unidades léxicas (Madrí, usté, verdá). Los hablantes que promocionan la elisión en el barrio de Salamanca (universitarios, clases medias-altas) tienen el prestigio social necesario para mantener la elisión en algunas unidades léxicas, pese a la tendencia normativa que les caracteriza.

En cambio, el ligero avance de la variante sorda $[\theta]$ en el barrio revela un intento de cambio desde abajo que no ha prosperado. Los hablantes madrileños que primero la adoptaron procedían de los niveles más bajos -el pueblo bajo madrileño, en palabras de Navarro Tomás-. La dental sorda ascendió socialmente durante los años 1970-1990 y consiguió penetrar en la clase media, especialmente a través de las mujeres jóvenes, pero no alcanzó los niveles sociales altos de la ciudad, lo que explicaría su escasa presencia en el barrio de Salamanca. A juzgar por el impulso que recibió la dental sorda durante los años de la transición, debía gozar de prestigio encubierto entre los niveles populares, un prestigio que no fue suficiente para lograr su ascenso social y su difusión entre los niveles altos.

La dental sonora [ð] representa el relevo generacional. Los hablantes más jóvenes no imitan a la generación anterior aceptando la pronunciación sorda de refuerzo. Su pronunciación oscila entre la elisión y la pronunciación normativa: aquella para usos coloquiales y familiares, esta para la pronunciación más cuidada de acuerdo con las pautas de prestigio abierto que la generación más joven aprendió en la escuela. De alguna manera, los 
jóvenes optan por la convergencia con las hablas meridionales en el habla informal y por la norma castellana en el habla formal. Se apartan, así, de la confluencia con las hablas del norte que había tratado de impulsar la generación anterior.

La baja actividad de la innovación en el barrio de Salamanca dificulta el seguimiento del cambio a través del espacio léxico, pues todas las tendencias, salvo la elisión, están muy poco marcadas, apenas apuntadas. Todas las unidades léxicas que se han estudiado independientemente: Madrid, ciudad, verdad, usted, edad, pueden realizarse con cualquiera de las tres variantes, sin embargo el análisis muestra que hay preferencias en cada caso por alguna de las tres soluciones:

[Ø]: verdad > verdá;

$[ð]:$ Madrid, usted $>$ Madrid, usted

[0]: edad, ciudad > ciudaz, edaz

El análisis permite ordenar en cada unidad léxica las preferencias por cada una de las tres variantes:

(1) verdá, (2) verdaz, (3) verdad;

(1) Madrid, (2) Madrí, (3) Madriz;

(1) usted, (2) usté, (3) ustez;

(1) edaz, (2) edá, (3) edad;

(1) ciudaz, (2) ciudá, (3) ciudad

Esta diferente distribución de soluciones por unidades léxicas avala la hipótesis de Navarro Tomás, según la cual, cada palabra tiene sus propios condicionantes y está sujeta a distintas restricciones que explican la falta de sincronía en el desarrollo léxico de la innovación. En la comunidad del barrio de Salamanca el seguimiento del cambio ha sido tan escaso que las distintas preferencias fónicas para cada unidad léxica deben entenderse en el contexto de una tendencia general a la elisión.

Por último, la comparación entre las hablas rurales madrileñas y el barrio de Salamanca de Madrid permite una mejor contextualización de la situación que hasta aquí se ha descrito. A partir de los datos rurales, sabemos que hubo un cambio de tendencia en el paso de la tercera a la segunda generación por parte de los niveles sociales y de instrucción bajos, que consistía en retroceder en las soluciones innovadoras meridionales en convergencia con el refuerzo dental sordo. Esta tendencia estaba liderada por los hombres de la segunda generación, pero no caló con la misma fuerza entre los jóvenes, quienes pese a imitar parcialmente a sus padres, retrocedieron en su uso de la interdental sorda, sustituyéndola en parte por la variantes sonora normativa. 
Este retroceso de los jóvenes fue más acusado entre las mujeres que entre los hombres.

En el barrio de Salamanca encontramos un "eco lejano" de la tendencia rural, si bien se puede afirmar que los madrileños del barrio de Salamanca no se han sumado al cambio intergeneracional que reflejan los hablantes rurales. Los parámetros de prestigio encubierto en el barrio, claramente a favor de la elisión, han frenado el cambio desde abajo, al tiempo que han mantenido las formas tradicionales de la comunidad. Solo las mujeres de la segunda generación hicieron un amago de incorporar la innovación, mostrando un ligero aumento de las soluciones sordas, pero ese intento ha tenido un impacto menor en el barrio de Salamanca.

\section{REFERENCIAS BIBLIOGRÁFICAS}

García Mouton, Pilar e Isabel Molina Martos. 2009. Trabajos sociodialectales en la Comunidad de Madrid. Revista de Filología Española LXXXIX / 1: 175-186. 2015a. Atlas Dialectal de Madrid. Madrid, CCHS-CSIC (adim.cchs.csic.es). 2015b. La -/d/ en el Atlas Dialectal de Madrid (ADiM): un cambio en marcha.

Lapurdum XIX: 277-290.

2015. La -/d/ en el Atlas Dialectal de Madrid (ADiM): un cambio en marcha. Lapurdum XIX: 277-290.

Molina Martos, Isabel y Florentino Paredes. 2014. Sociolingüística de la elisión de la dental -/d/- en Madrid (distrito de Salamanca). Cuadernos de Lingüistica del Colegio de México. Estudios de cambio y variación 2: 55-114. <http://cuadernoslinguistica.colmex.mx/>

Molina Martos, Isabel. 2015. La variable sociolingüística -/s/ en el distrito de Vallecas (Madrid). Patrones sociolingüísticos de Madrid, pp. 91-115. Frankfurt am Main, Peter Lang. Fondo Hispánico de Lingüística y Filología.

NAVArRo Tomás, Tomás. [1918] 1981, 21. (ed.). Manual de pronunciación española. Madrid, CSIC.

1975. Áreas geográficas de consonantes finales. Capitulos de geografía lingüística de la Península Ibérica. Bogotá, Instituto Caro y Cuervo.

SAnkoff, D., TAgliamonte, S., \& Smith, E. 2012. Goldvarb LION: A variable rule application for Macintosh. University of Toronto. URL http://individual. utoronto. ca/tagliamonte/ goldvarb. htm 